\title{
Détermination des sites de réflexion : localisation d'une jonction entre deux conduites cylindriques
}

\author{
Fatima Chirazi, JaÂfar Khalid Naciri et Mohamed Ouazzani Touhami \\ Université Hassan II, Faculté des sciences Aïn Chock, Laboratoire de Mécanique, km 8, Route d'El Jadida, BP 5366 Maârif, \\ Casablanca, Maroc
}

Reçu le 2 juin 2003, accepté le 5 mars 2004

\begin{abstract}
Résumé - Dans ce papier, nous élaborons une technique d'interprétation des ondes réfléchies. Cette technique est basée sur une approche analytique permettant de maîtriser la propagation pure d'une onde incidente et par suite d'interpréter toute information transportée par l'onde réfléchie. L'objectif essentiel de cette démarche est de développer une méthodologie pour la détermination des sites de réflexions telles que la localisation d'une jonction avec ou sans obstruction.
\end{abstract}

Mots clés : Propagation d'ondes / évolution d'un signal émis à l'extrémité / sites de réflexion / jonction / sténose

\begin{abstract}
Determining the reflection sites: Localisation of a junction between two cylindrical conduit. In this paper, we develop an technical interpretation of reflected waves. This technique is based on an analytical approach in order to control the pure of the incident wave and then interpret any transported information by reflected wave. Primarily we present a methodology for determining the reflection site such as the jonction localization with or without obstruction.
\end{abstract}

Key words: Wave propagation / signal emitted at the extremity / reflection sites / jonction / stenosis

\section{Nomenclature}

\begin{tabular}{|ll|}
\hline$A$ & section du tube $\left(\mathrm{m}^{2}\right)$ \\
$A_{\mathrm{s} n}$ & section de référence $\left(\mathrm{m}^{2}\right)$ \\
$L_{n}$ & longueur du tube $(\mathrm{m})$ \\
$P$ & pression $\left(\mathrm{kg} \cdot \mathrm{s}^{-2} \cdot \mathrm{m}^{-1}\right)$ \\
$P_{\mathrm{s} n}$ & pression de référence $\left(\mathrm{kg} \cdot \mathrm{s}^{-2} \cdot \mathrm{m}^{-1}\right)$ \\
$Q$ & débit $\left(\mathrm{m}^{3} \cdot \mathrm{s}^{-1}\right)$ \\
$Q_{\mathrm{i} n}$ & débit initial $\left(\mathrm{m}^{3} \cdot \mathrm{s}^{-1}\right)$ \\
$t$ & temps $(\mathrm{s})$ \\
$z$ & cordonnée axial $(\mathrm{m})$ \\
$V_{\mathrm{o} n}$ & vitesse de propagation $\left(\mathrm{m} \cdot \mathrm{s}^{-1}\right)$ \\
$\rho$ & masse volumique du fluide $\left(\mathrm{kg} \cdot \mathrm{m}^{-3}\right)$ \\
\hline
\end{tabular}

\section{Introduction}

Dans un réseau de conduites déformables, la détermination des sites de réflexion, de nature géométrique ou mécanique, constitue l'un des principaux objets de l'étude du phénomène de propagation des ondes de pression, de débit ou de section dans ce réseau. Toutefois et malgré la multitude des méthodes mises en œuvre

\footnotetext{
a Auteur correspondant : touazzani@hotmail.com
}

pour cette détection, il apparaît clairement qu'il n'est pas toujours systématique de déduire, à partir de l'analyse des changements observés dans l'allure d'un signal émis à l'extrémité de ce réseau une information complète sur ces sites de réflexion. Les résultats théoriques et expérimentaux, déjà établis dans ce cadre $[1,2]$ permettent, lorsqu'il s'agit d'un tube fermé à son extrémité avale, de suivre l'évolution d'une forme donnée d'un signal émis à l'entrée du tube et par la suite d'accéder aux différentes informations relatives aux sites de réflexion à partir d'une simple interprétation des ondes réfléchies. Cette façon de faire est, par contre, non évidente lorsqu'il s'agit d'un tube qui est déjà le siège d'un écoulement existant à l'instant d'émission d'un signal spécifique à son entrée. Pour ce cas, où on voit apparaître, dès l'instant initial, un phénomène de réflexion à l'entrée du tube, il devient, en effet, difficile de distinguer, dans les réflexions obtenues, celles liées au signal émis de celles qui existeraient en son absence.

Dans ce travail, on propose une approche purement analytique permettant d'accéder à une interprétation rigoureuse des ondes réfléchies et par la suite d'établir une corrélation entre celles-ci et la position des sites de réflexion. Nous élaborons en particulier une méthodologie pour la localisation d'une jonction dans ce réseau. 


$$
f_{1}(s)=\left\{\begin{array}{rr}
-\frac{1}{2 V_{\mathrm{o} 1}} Q_{\mathrm{i} 1}\left(s-2 k L_{1}\right)+\sum_{\substack{m=0 \\
k \succ m}}^{k-1} a_{1} B_{1}\left[\frac{s-(2 m+1) L_{1}}{V_{\mathrm{o} 1}}\right]-\sum_{\substack{m=1 \\
k \geq m}}^{k} a_{0} H\left[\frac{s-2 m L_{1}}{V_{\mathrm{o} 1}}\right]+C_{j 1} \\
\left.-\frac{1}{2 V_{\mathrm{o} 1}} Q_{\mathrm{i} 1}\left((2 k+2) L_{1}-s\right)+\sum_{m=0}^{k} a_{1} B_{1}\left[\frac{s-(2 m+1) L_{1}}{V_{\mathrm{o} 1}}\right]-\sum_{\substack{m=1 \\
k \geq m}}^{k} a_{0} H\left[\frac{s-2 m L}{V_{\mathrm{o} 1}}\right]+C_{j 1},(2 k+1) L_{1}\right] \\
& \forall s \in\left[(2 k+1) L_{1},(2 k+2) L_{1}\right]
\end{array}\right.
$$

$$
g_{1}(s)= \begin{cases}A_{\mathrm{s} 1}-f_{1}(-s)+a_{0} H\left(-\frac{s}{V_{\mathrm{o} 1}}\right) & \underline{\forall s \leq 0} \\ A_{\mathrm{s} 1}-f_{1}(s) & \underline{\forall s \in\left[0, L_{1}\right]}\end{cases}
$$

\section{Formulation}

On considère l'écoulement unidimensionnel d'un fluide non visqueux dans un réseau de deux tubes raccordés à parois souples, axisymétriques de longueurs $L_{n=1,2}$ et d'axe $z$.

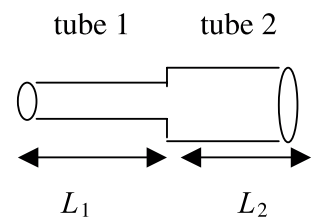

Schéma représentatif de la jonction considérée.

Le comportement mécanique de la paroi est pris en compte à travers la relation pression section introduite par Rockwell [3] :

$$
A_{n}(z, t)=A_{\mathrm{s} n} \exp \left[\xi_{n}\left(P_{n}(z, t)-P_{\mathrm{s} n}\right) / A_{\mathrm{s} n}\right] \quad n=1,2
$$

où $A_{\mathrm{s} n}$ et $P_{\mathrm{s} n}$ représentent respectivement une section et une pression de référence et $\xi_{n}$ une constante qui caractérise la rigidité du tube considéré.

Dans ces conditions, et dans le cas où les termes convectifs sont négligeables, la forme unidimensionnelle des équations globales de conservation de la masse et de la quantité de mouvement, se ramènent à l'équation de propagation, en terme de section, suivante :

$$
\frac{\partial^{2} A_{n}}{\partial t^{2}}-V_{\mathrm{o} n}^{2} \frac{\partial^{2} A_{n}}{\partial z^{2}}=0 \quad n=1,2
$$

où $V_{\mathrm{o} n}=\sqrt{A_{\mathrm{s} n} / \rho \xi_{n}}$ est la vitesse de propagation.

Notons qu'il est possible d'obtenir, dans ces mêmes conditions, une équation de propagation, en terme de débit, similaire à l'équation (2) et avec la même vitesse de propagation.

Pour ce problème les conditions aux limites et initiales considérées sont définies par :

$$
\begin{gathered}
A_{1}(z=0, t)=A_{\mathrm{s} 1}+a_{0} H(t), A_{2}\left(z=L_{2}, t\right)=A_{\mathrm{s} 2} \\
\forall t \geq 0 \\
\begin{array}{c}
A_{n}(z, t=0)=A_{\mathrm{s} n}, Q_{n}(z, t=0)=Q_{\mathrm{i} n}(z) \\
\forall z \in\left[0, L_{n}\right]
\end{array}
\end{gathered}
$$

où $H(t)$ est une fonction connue du temps représentant le signal émis à l'extrémité amont de la jonction, $a_{0}$ son amplitude et $Q_{n}(z, t)$ le débit. Les quantités $A_{\mathrm{s} n=1,2}, L_{n=1,2}$ et $Q_{\mathrm{in} n=1,2}(z)$ désignent respectivement les sections au repos, les longueurs et les débits initiaux dans les tubes 1 et 2 de la jonction.

Pour procéder à une étude analytique, similaire à celle que nous avons déjà développée dans un travail précédent [4], du phénomène de propagation des ondes de section dans chacun des deux tubes, on pose :

$$
\begin{aligned}
& A_{1}\left(z=L_{1}, t\right)=A_{\mathrm{s} 1}+a_{1} B_{1}(t), \\
& A_{2}(z=0, t)=A_{\mathrm{s} 2}+a_{2} B_{2}(t) \quad \forall t \geq 0
\end{aligned}
$$

où $B_{n=1,2}$ sont des fonctions inconnues. Dans ce cas, la compatibilité entre les conditions initiales et aux limites pour chacun des tubes est assurée à travers les relations suivantes :

$$
\begin{array}{ll}
Q_{\mathrm{i} 1}^{\prime}(0)=-a_{0} H^{\prime}(0), & Q_{\mathrm{i} 1}^{\prime}\left(L_{1}\right)=-a_{1} B_{1}^{\prime}(0) \\
Q_{\mathrm{i} 2}^{\prime}(0)=-a_{2} B_{2}^{\prime}(0), & Q_{\mathrm{i} 2}^{\prime}\left(L_{2}\right)=0, \quad B_{n=1,2}(0)=0
\end{array}
$$

$Q_{\text {in }}^{\prime}$ sont les dérivées, par rapport à $z$, des débits initiaux et $B_{n}^{\prime}$ les dérivées, par rapport au temps, des fonctions $B_{n}$.

\section{Résolution analytique}

Dans le cadre de notre formulation, la solution de l'équation (2) pour chaque tube de la jonction se présente sous la forme :

$$
A_{n}(z, t)=f_{n}\left(z+V_{\text {on }} t\right)+g_{n}\left(z-V_{\text {on }} t\right) \quad n=1,2
$$

$f_{n}$ et $g_{n}$ sont respectivement les composantes réfléchies et incidentes dans chaque tube de la jonction que nous déterminons en tenant compte des conditions (3-5). On obtient après calculs [4] :

- tube 1

voir l'équation au-dessus 


$$
f_{2}(s)=\left\{\begin{aligned}
-\frac{1}{2 V_{\mathrm{o} 2}} Q_{\mathrm{i} 2}\left(s-2 k L_{2}\right)-\sum_{\substack{m=1 \\
k \geq m}}^{k} a_{2} B_{2}\left[\frac{s-2 m L_{2}}{V_{\mathrm{o} 2}}\right] & +C_{j 2} \\
& \frac{\forall s \in\left[2 k L_{2},(2 k+1) L_{2}\right]}{{ }^{2} V_{\mathrm{o} 2}} Q_{\mathrm{i} 2}\left((2 k+2) L_{2}-s\right)-\sum_{\substack{m=1 \\
k \geq m}}^{k} a_{2} B_{2}\left[\frac{s-2 m L_{2}}{V_{\mathrm{o} 2}}\right] \\
& \underline{\forall s \in\left[(2 k+1) L_{2},(2 k+2) L_{2}\right]}
\end{aligned}\right.
$$

$$
g_{2}(s)= \begin{cases}A_{\mathrm{s} 2}-f_{2}(-s)+a_{2} B_{2}\left(-\frac{s}{V_{\mathrm{o} 2}}\right) & \underline{\forall s \leq 0} \\ A_{\mathrm{s} 2}-f_{2}(s) & \underline{\forall s \in\left[0, L_{2}\right]}\end{cases}
$$

- tube 2

voir l'équation au-dessus

où $s$ est un argument qui vaut $z+V_{\text {on }} t$ ou $z-V_{\text {on }} t$ suivant qu'il s'agisse de la fonction $f_{n=1,2}$ ou $g_{n=1,2}, C_{j n=1,2}$ sont des constantes arbitraires et $k$ le nombre des aller-retours de l'onde.

À partir de ces expressions, nous constatons que les fonctions $f_{n=1,2}$ et $g_{n=1,2}$ dépendent de la perturbation $H(t)$ introduite à l'extrémité amont du tube 1 , des conditions initiales $Q_{\mathrm{i} n=1,2}(z)$ associées à l'écoulement dans la jonction et des fonctions $B_{n=1,2}$ qui sont jusqu'à présent inconnues.

Par ailleurs, la connaissance des fonctions $f_{n}$ et $g_{n}$ permet d'accéder à l'expression du débit $Q_{n}(z, t)$ dans chaque tube; celui-ci est, en effet, égal à :

$$
Q_{n}(z, t)=-V_{\text {on }}\left[f_{n}\left(z+V_{\text {on }} t\right)-g_{n}\left(z-V_{\text {on }} t\right)\right]+e_{n}
$$

où $e_{n}$ est une constante d'intégration qui dépend des conditions initiales de notre problème.

La détermination des fonctions $B_{n}$ nécessite un traitement particulier à la jonction. Pour se faire nous supposons que la perte de charge à la section de raccord est nulle. Dans ce cadre, la condition traduisant la continuité de la pression de part et d'autre de la jonction permet, compte tenu des équations (1) et (4), d'aboutir à :

$$
\left[1+\frac{a_{2}}{A_{\mathrm{s} 2}} B_{2}(t)\right]=\left[1+\frac{a_{1}}{A_{\mathrm{s} 1}} B_{1}(t)\right]^{d}
$$

Par la suite, et pour l'obtention d'une deuxième condition portant sur ces fonctions, il est suffisant de traduire, à partir de la solution en terme de débit et des expressions des fonctions $f_{n}$ et $g_{n}$ pour chaque tube, la condition de conservation de débit à la traversée de la jonction.

Ainsi, et dans le cas où on se limite à un seul allerretour de l'onde $(k=1)$, cette condition se ramène à :

$$
\left[1+\frac{a_{2}}{A_{\mathrm{s} 2}} B_{2}(t)\right]=p_{\mathrm{o}}-p_{1}\left[1+\frac{a_{1}}{A_{\mathrm{s} 1}} B_{1}(t)\right]+p_{2}(t)
$$

avec $: d=\left(V_{\mathrm{o} 1} / V_{\mathrm{o} 2}\right)^{2}$

$$
\begin{gathered}
p_{\mathrm{o}}=1+\frac{V_{\mathrm{o} 2} A_{\mathrm{s} 1}}{V_{\mathrm{o} 1} A_{\mathrm{s} 2}}-\frac{a_{0} V_{\mathrm{o} 2}}{A_{\mathrm{s} 2}} H^{\prime}(0)\left(\frac{L_{1}}{2}-L_{2}\right) \\
p_{1}=\frac{V_{\mathrm{o} 2} A_{\mathrm{s} 1}}{V_{\mathrm{o} 1} A_{\mathrm{s} 2}} \\
p_{2}(t)=\frac{a_{0}}{2 L_{1}} \frac{V_{\mathrm{o} 2}}{A_{\mathrm{s} 2}} H^{\prime}(0)\left[L_{1}^{2}-2 V_{\mathrm{o} 1} t L_{1}+2\left(V_{\mathrm{o} 1} t\right)^{2}\right] \\
+\frac{a_{0}}{L_{1}} \frac{V_{\mathrm{o} 2}}{A_{\mathrm{s} 2}} H^{\prime}(0)\left[V_{\mathrm{o} 1} t-L_{2}\right]^{2}
\end{gathered}
$$

La résolution du système d'équations (6) et (7) permet alors de déduire les fonctions $B_{n=1,2}$ pour lesquelles les relations de compatibilités précédentes sont vérifiées.

\section{Résultats et conclusion}

La solution analytique proposée permet de distinguer clairement dans les expressions des ondes réfléchies $\left(f_{n}\right)$ la part liée au signal émis à l'extrémité du tube 1 et la part liée à l'initialisation imposée. En effet, l'expression de $f_{n}$ montre que dans le cas où on considère $H=0\left(\right.$ et $\left.B_{n}=0\right)$ c'est-à-dire en absence d'un signal émis aux extrémités de chaque tube de la jonction, il existe encore une composante réfléchie à travers les termes $-Q_{\mathrm{i} n}\left(s-2 k L_{n}\right)$ pour $s \in\left[2 k L_{n},(2 k+1) L_{n}\right]$ et $-Q_{\text {in }}\left[(2 k+2) L_{n}-s\right]$ pour $s \in\left[(2 k+1) L_{n},\left(2 k+12 L_{n}\right]\right.$ ce qui produit un phénomène de réflexion dès l'instant initial à l'entrée de la jonction. Cette réflexion initiale se superpose donc à celle du signal émis et rend difficile l'interprétation des ondes réfléchies obtenues. L'originalité de la solution analytique réside alors dans le fait qu'elle permet d'extraire, à partir des expressions de $f_{n}$, cette réflexion initiale et par conséquent 


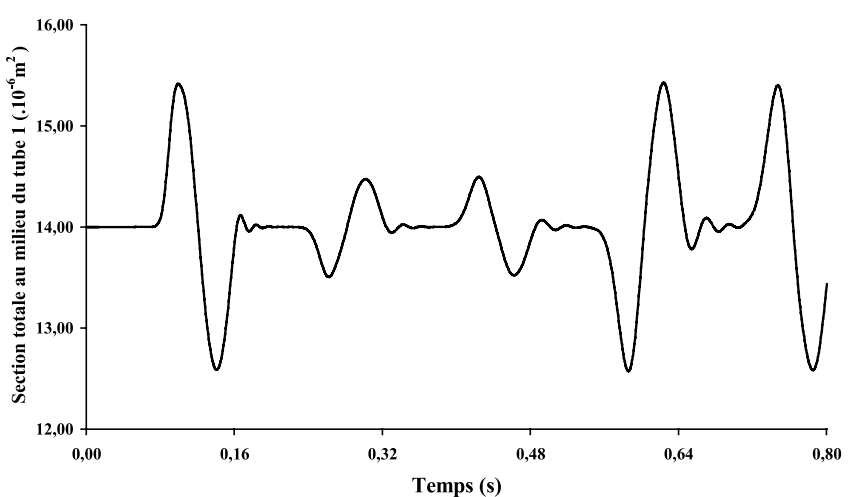

Fig. 1. Évolution de la section totale au cours du temps pour $H(t)=\sin (2 \pi t / 0,08), L_{1}=L_{2}=0,25 \mathrm{~m}, A_{\mathrm{s}}=14 \times 10^{-6} \mathrm{~m}^{2}$, $A_{\mathrm{s}}=28 \times 10^{-6} \mathrm{~m}^{2}, \xi_{1}=55 \times 10^{-10} \mathrm{~cm}^{4}$. dyne $^{-1}, \xi_{2}=110 \times$ $10^{-10} \mathrm{~cm}^{4}$.dyne ${ }^{-1}$.

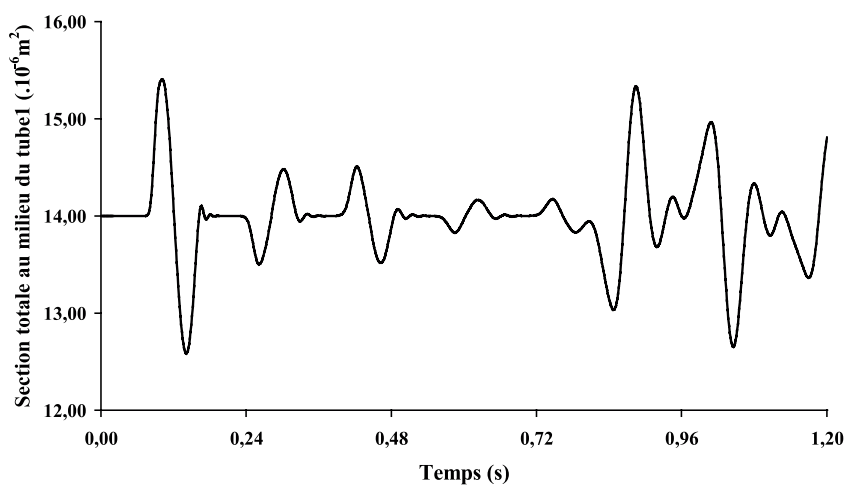

Fig. 2. Évolution de la section totale au cours du temps pour $H(t)=\sin (2 \pi t / 0,08), L_{1}=0,25 \mathrm{~m}, L_{2}=0,45 \mathrm{~m}, A_{\mathrm{s}}=$ $14 \times 10^{-6} \mathrm{~m}^{2}, A_{\mathrm{s}}=28 \times 10^{-6} \mathrm{~m}^{2}, \xi_{1}=55 \times 10^{-10} \mathrm{~cm}^{4}$.dyne ${ }^{-1}$, $\xi_{2}=110 \times 10^{-10} \mathrm{~cm}^{4}$.dyne ${ }^{-1}$.

d'analyser et d'interpréter rigoureusement toute information transportée par le signal réfléchi. Cette interprétation constitue un moyen privilégié pour la caractérisation des sites de réflexion de l'onde.

Ainsi, et pour le cas particulier d'une onde solitaire pour laquelle la durée de l'excitation initiale reste suffisamment faible par rapport à $L_{1} / V_{\mathrm{o} 1}$ (ce qui permet à l'onde de se réfléchir à la rencontre de la jonction), l'analyse de ce phénomène de propagation pour un seul aller retour $(k=1)$ est suffisante pour distinguer en un site donné la phase aller du signal de sa phase retour.

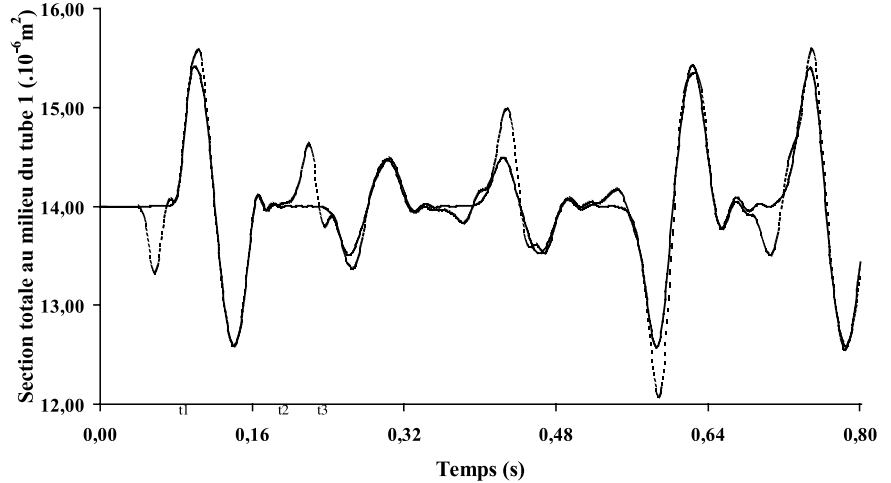

Fig. 3. Évolution de la section totale au cours du temps pour $H(t)=\sin (2 \pi t / 0,08)$ si $0 \leq t \leq 0,08$ et 0 si $t \geq 0,08 . L_{1}=$ $L_{2}=0,25 \mathrm{~m}, A_{\mathrm{s}}=14 \times 10^{-6} \mathrm{~m}^{2}, A_{\mathrm{s}}=28 \times 10^{-6} \mathrm{~m}^{2}, L_{\mathrm{s}}=$ $0,04 \mathrm{~m}, \delta=0,1$.

Diverses applications peuvent alors être envisagées à partir de cette propriété notamment la localisation et la caractérisation de la jonction et d'une sténose présente dans celle-ci. Pour cette application, la mesure de la durée qui sépare les passages successifs du signal dans une section donnée de la jonction est suffisante pour déterminer la longueur de chaque tube et par conséquent localiser la jonction (Figs. 1, 2) et la sténose (Fig. 3).

\section{Références}

[1] F. Pythoud, N. Stergiopulos, N. Westerhof, J.J. Meister, Method for determining distribution of reflection sites in the arterial system, Am. J. Physio. 271 (1996) H1807-H1813

[2] A. Ghouli, J. Khalid Naciri, M. Zagzoule, M. Ouazzani Touhami, Méthode de séparation d'ondes périodiques pour les écoulements de fluide visqueux en conduite souple : cas non linéaire, C. R. Acad. Sci. Paris, Série IIb 325 (1997) 61-68

[3] R.L. Rockwell, Non-linear analysis of pressure and shock waves in blood vessels, Ph.D. Stanford University, 1969

[4] F. Chirazi, J. Khalid Naciri, M. Ouazzani Touhami, Étude analytique de la propagation d'ondes en conduites déformables, C. R. Acad. Sci. Paris, Série IIb 326 (1998) 845-850 